\title{
Locality Detection in Wireless Sensor Network using Population based Algorithm
}

\author{
Avinash Kaur \\ Department of CSE, \\ CSVTU, Bhilai, India
}

\author{
Sonu Agrawal \\ Department of CSE, \\ CSVTU, Bhilai, India
}

\begin{abstract}
Artificial Bee Colony Algorithm is a population based optimization method depends on the behaviour of natural honey bees. It works on the concept of natural bees for finding nectar food source. In this we show how $A B C$ is applied for the application of location detection in wireless environment. Wireless Sensor Networks (WSN) is used for hunt down targets, environmental supervising etc. While these networks are widely used in many applications, their success highly depends on the sensor node positions known as network deployment. Determining the position of sensor nodes is the main objective of deployment network which directly depends on the coverage of the concerned region. To locate sensors, Global Positioning System (GPS) is also used by which sensors know their position. But, this method is not feasible due to economic issues. So, only a small part of the network can affordably be equipped with GPS, and an automatic localization process is required for the rest of the nodes in the network. We guess random solutions and then apply some method to find the best possible solution.
\end{abstract}

\section{KEYWORDS}

Wireless Sensor Network (WSN), Location Discovery (LD) Multilateration, Artificial Bee Colony (ABC)

\section{INTRODUCTION}

In recent years, optimization and wireless sensor networks become a latest topic in research. ABC technique is very efficient in cases where the assumptions are fulfilled correctly. A branch of nature inspired algorithms which are called as swarm intelligence is focused on insect behavior in order to develop some meta-heuristics which can mimic insect's problem solution abilities. Interaction between insects contributes to the collective intelligence of the social insect colonies. These communication systems between insects have been adapted to scientific problems for optimization.WSN generally have capabilities for monitoring hefty area, fire fighting, transportation tracking etc. One of the applications of WSN is location discovery. To know the exact location of an object we use sensors. We can give GPS to each node. But, it may not be suitable for movable users due to its high power consumptions. Therefore, we can give GPS to a petite subset of nodes known as beacon nodes and evaluate the distance of other nodes with reference to beacon nodes [1]. Many localization techniques are used to measure the distance between sensor nodes. Firstly, nodes are localized using measurements like distance, time of arrival (ToA), time difference of arrival (TDoA), angle of arrival (AoA), direction of arrival. Then, position computation techniques i.e., $\mathrm{ABC}$ algorithm is used to compute position of a node, and refining its value [2].

However, the distance estimated contains error which ranges from slight to larger ones. The main aim of LD is to minimize the distance estimated error [3]. In this paper, we propose Artificial Bee Colony Algorithm for location detection in Wireless Sensor Networks \& compare it with the estimated values to find the effectiveness of $\mathrm{ABC}$.

\section{LOCATION DISCOVERY}

The task of determining the node locations using distances between two nodes which combined with a set of known node locations is referred to as location discovery (LD). The main drawback found in LD is the presence of distance assessment errors, which result in node positioning errors [4-5]. Location Discovery problem is the type of optimization problem where we have to find a set of solutions in such a way so that location error is minimized. Here, we consider an optimization technique which is used to find the location of a node with respect to seven beacon nodes and 63 unknown nodes in a $(300,300)$ distance area as shown in Fig. 1

$(0,300)$

$(300,300)$

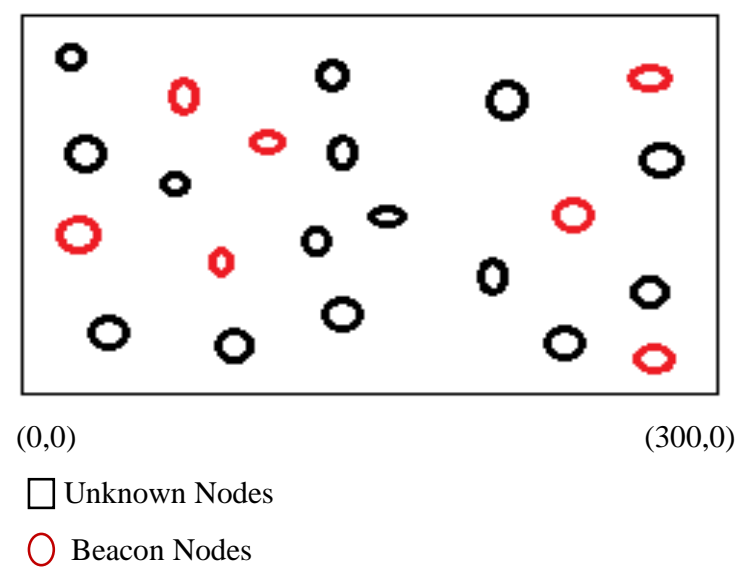

Figure 1: Multilateration System

\section{WIRELESS SENSOR NETWORK}

Wireless Sensor Networks are an emerging technology that has been used in various applications. It monitors the physical world using small wireless devices known as sensor nodes [6]. Wireless Sensor Network localization techniques are used to locate sensor nodes with the support of reference nodes and beacon nodes. Beacon Nodes are those who know their position coordinates [7]. Other nodes are localized using measurements like distance, time of arrival (ToA), time difference of 
arrival (TDoA), angle of arrival (AoA), direction of arrival (DoA)

Then, position computation techniques like multilateration are used to compute position of a node. Finally, optimization algorithms are used to locate WSN nodes. Several application scenarios of WSNs are present which include military, industrial, household, medical, natural disasters monitoring, and other emergency situations.

\section{PROPOSED ARTIFICIAL BEE COLONY TECHNIQUE}

The target of the proposed approach is to estimate the position of nodes in a wireless environment with minimum error.

Optimizations are typically performed in an iterative trend as shown in Figure 2.



Figure 2:-The Optimization Steps

The hunt is started either from one or more random solutions chosen according to some decisive factor. In the evaluation stage, the quality (fitness) of the current locations(s) is calculated by experiments [8-9].

ABC algorithm contains three types of bees: Employed Bee, Onlooker Bee, and Scout Bee [10]. A bee go to the food source which she previously visited is known as Employed Bee. A bee waiting on the dancing area for choosing food source is known as onlooker bee. A bee goes and find new food source by random search is known as Scout Bees.

In $\mathrm{ABC}$ algorithm, initially a food source position is randomly selected by the bees \& their nectar amount is determined. Then these bees go to their hives and distribute this information to other bees. At next stage, the employed bees goes to the food source area visited by them in the previous cycle and find a new food source in the neighbor of their present location. At the last stage, an onlooker bee goes to the area where the nectar amount of food is higher compare to a fixed estimated value. When she arrives at the selected area she chooses a new food source by visualizing in its neighborhood. Visual information is depending on the comparison of food source position. When the food is deserted by the bees, a new food source is arbitrarily determined by a scout bee [1213].

In ABC algorithm, the nectar amount of food determines the fitness or quality of a food source and the positions of food source correspond to solution of the optimization problem.

The number of employed bee and the onlooker bee is equals to the half of the food source or colony size.

In this, at the first stage $\mathrm{ABC}$ generates randomly generate initial population of $\mathrm{N}$ size. Each solution is a D-dimension vector, where $\mathrm{D}$ is the optimization parameters. After this, the population of solution is subjected to repeated cycles $(C=1,2,3 \ldots 2000)$ of search processes of the employed bees, onlooker bees
$\&$ scout bees [14]. An employed or onlooker bee produces a modification on the position in her memory for finding a new food source \& tests the fitness value of the new food source.

The production of the new food source position is also based on a comparison process of food source positions. They randomly select a food source position \& produce a modification on the existing in their memory as

$B_{i j}=x_{i j}+\alpha_{i j}\left(x_{i j}+x_{k j}\right)$

Where $\mathrm{k}=[1,2 \ldots$ Beacon node $]$ chosen randomly and $\alpha_{\mathrm{ij}}$ is a random number between $[-1,1]$. It controls the position of neighbor food source around $\mathrm{x}_{\mathrm{ij}}$. For each candidate solution of a food source $B_{i j}$ is evaluated and compared with the old value. If the nectar amount of new food source is higher than the previous one, the bee memorizes the new position \& forgets the old one. After the search process of employed bees completes, they share this information $\&$ their position information with the onlooker bees on dancing area [15]. An onlooker bee evaluates the nectar information \& chooses a food source with a probability related to its nectar amount. An onlooker bee selects a food source depending on the probability value associated with that food source, $\mathrm{PR}_{\mathrm{i}}$ by,

$$
\begin{array}{r}
\mathrm{N}=\mathrm{N} \\
\mathrm{PR}_{\mathrm{i}}=\mathrm{fit}_{\mathrm{i}} / \sum \mathrm{fit}_{\mathrm{n}} \\
\mathrm{n}=1
\end{array}
$$

Where fit $_{\mathrm{i}}$ is the fitness value of the solution evaluated by employed bee $\& \mathrm{~N}$ is the number of food sources.

In order to produce a candidate food position from the old one, the $\mathrm{ABC}$ uses the following expression (3):

$$
\mathrm{v}_{\mathrm{ij}}=\mathrm{x}_{\mathrm{ij}}+\varphi_{\mathrm{ij}}\left(\mathrm{x}_{\mathrm{ij}}-\mathrm{x}_{\mathrm{kj}}\right) \text {, }
$$

Where $k \in\{1,2 \ldots . \mathrm{BN}\}$ and $\mathrm{j} \in\{1,2 \ldots \mathrm{D}\}$ are randomly chosen indexes. Although $\mathrm{k}$ is determined randomly, it has to be different from i. $\varphi_{\mathrm{i}, \mathrm{j}}$ is a random number between $[-1,1]$. It controls the production of a neighbour food source position around $\mathrm{x}_{\mathrm{i}, \mathrm{j}}$ and the modification represents the comparison of the neighbour food positions visually by the bee [16]. This process continues until the nectar food is abandoned.

\section{PROPOSED METHODOLOGY}

The aim of the proposed approach is to estimate the positions of wireless nodes spread in a $2 \mathrm{D}$ space with the help of the calculated ranges of each unknown node to $\mathrm{N}$ reference nodes with known positions. In this paper, $A B C$ algorithm is applied for finding the location of unknown nodes in a wireless environment with the help of beacon nodes. Beacon nodes are the nodes whose $\mathrm{x}$, $\mathrm{y}$ coordinates are known. In this at first stage, we randomly choose the positions of unknown nodes by some measuring technique. Then, at second stage employed bees goes to the position of unknown nodes and modified it using equ. (1), at the third stage, onlooker bee goes and updates the fitness value obtained by the employed bee. Employed bee modify all the distance measured for each unknown node but Onlooker bee only updates the distance of particular 
candidate solution depending upon the probability associated with that food source as in equa.(2) .

If a parameter produced by this operation exceeds its predetermined limit, the parameter can be set to a satisfactory value. In this work, the value of the parameter greater than its limit is set to its limit value. In $A B C$ algorithm, if a position cannot be enhanced further through a predetermined quantity of cycles called limit then that solution is assumed to be deserted.

\section{RESULT}

The $A B C$ algorithm is applied to solve the localization problem in a $2 \mathrm{D}$ space, to 63 unknown nodes and 7 reference nodes, with 2000 cycles. The mean value and standard deviation got is:-

Mean $(\mathrm{m})=53451.9$

Standard Deviation $(\mathrm{SD})=938.919$

\section{CONCLUSION AND FUTURE RESEARCH DIRECTION}

Sensor location is vital for many critical applications like battlefield surveillance, target tracking, environmental monitoring, wildfire detection, and traffic regulation [10]. Location discovery is still an active area of research, both in terms of proposing and validating specific applications and in terms of developing a theoretical framework. Localization has two important metrics: efficiency and accuracy [11]. In this, we have discussed the unique operational challenges faced by WSNs, presented a comprehensive overview of the localization process, and discussed the optimization algorithm. In future, the work is going to improve this value and make it more accurate.

\section{REFERENCES}

[1] Guillermo Molina, Enrique Alba, "Location discovery in Wireless Sensor Networks using metaheuristics", Elsevier, 2010,pp. 1224-1240.

[2] D.Culler, D. Estrin, M. Srivastava, "Overview of sensor networks", IEEE Comput. 37 (8) (2004) 41-49.

[3] Guangjie Han,Huihui, Trung Q. Duong , Jinfang Jiang, Takahiro Hara "Localization algorithms of Wireless Sensor Networks: a survey" Springer(2011).

[4] Meisam Mappar and Amir Masoud Rahmani "A New Approach for Sensor Scheduling in Wireless Sensor Networks Using Simulated Annealing", Fourth International Conference on Computer Sciences and Convergence Information Technology, pp. 748-750, 2009.

[5] G. J. Pottie and W. J. Kaiser, "Wireless integrated network sensors ", Communications of the ACM, vol. 43, pp. 51-58, 2000.
[6] Hsin-Chih Wang, Men-Shen Tsai and Yu-Cheng Wang, "Performance comparition of genetic algorithm and artificial bee colony algorithm applications for localization in wireless sensor networks", ICSSE, pp. 469-474, 2010.

[7] Celal Ozturk, Dervis Karaboga and Beyza Gorkemli, "Probability dynamic deployment of wireless sensor networks by artificial bee colony algorithm", sensors, pp. 6056-6065, 2011.

[8] Moussa and N. El-Sheimy, "Localization of wireless sensor network using bees optimization algorithm", IEEE, pp. 478-481, 2011.

[9] J. Feng, L Girod, M. Potkonjak, "Location Discovery using data-driven statistical error modelling", IEEE Conference on computer communication , pp. 1-14, April 2006.

[10] M. L. Sichitiu and V. Ramadurai (2004), "Localization of wireless sensor networks with a mobile beacon", Proceedings of the 1st IEEE International Conference on Mobile $\mathrm{Ad}$ hoc and sensor systems, October, pp. 174-183, 2004.

[11] W. R. Heinzelman, A. Chandrakasan, and H. Balakrishnan, "Energy efficient communication protocols for wireless microsensor networks", HICSS, 2000.

[12] Dervis Karaboga, Bahriye Akay "A comparative study of Artificial Bee Colony algorithm" Elsevier (2009)

[13] Lei Zhang and Binwei Deng, "A New Range based Localization Algorithm for Wireless Sensor Networks", International Colloquium on Computing, Communication, Control, and Management (CCCM 2009), pp. 111-114.

[14] Christian P. Robert, Mark A Beaumont. Jean Michel Marin, "Adaptivity for $\mathrm{ABC}$ algorithms: the $\mathrm{ABC} P \mathrm{PMC}$ scheme", International Society for Bayesian Analysis (2008), pp. 1-14.

[15] D. Culler, D. Estrin, M. Srivastava, "Overview of sensor networks", IEEE Comput. 37 (8) (2004) 4149.

[16] Dervis Karaboga and Bahriye Basturk “ Artificial Bee Colony (ABC) Optimization Algorithm for Solving Constrained Optimization Problems "Springer(2007). 